\title{
Design and Implementation of Teaching Assistant System based on Web Development Technique for Advanced Mathematics
}

\author{
PANG Tong ${ }^{1, a}$ \\ ${ }^{1}$ Guangxi Technical College of Machinery and electricty, Nanning 530007, China \\ ajD3249466@126.com
}

Keywords: Teaching Assistant System; Advanced Mathematics; Web based Technique.

\begin{abstract}
In this paper, we theoretically research the implementation of teaching assistant system based on web development technique for advanced mathematics in this manuscript. Computer assisted learning method has already become a kind of very important teaching mode. Mathematics is established on the basis of the practical application of as early as one hundred years ago and Engels pointed out: mathematics is the study of quantitative relationship between the real world and the spatial form of science. Therefore, the combination of computer technique and math teaching is necessary. Our designed system will help the teachers to manage the teaching activity easier.
\end{abstract}

\section{Introduction}

With the constant and bursting development of computer science and technology, the computer and computer network are taken in important place. Computer assisted learning method has already become a kind of very important teaching mode. Many places have adopted at home and abroad based on microwave transmission and cable television network or network transmission of remote teaching methods but for a lot of units, microwave transmission and cable television network will need a huge technical and financial input, therefore it is difficult to popularization. For a long period of time, in the mathematics teaching in people's impression is the concept and people's knowledge of mathematics is a strict thinking of science and mathematics activity is considered to be highly abstract thinking activities [1-2] . In fact this is just one aspect of mathematics and in the modern life learning, mathematics has a wider applicability. Mathematics is established on the basis of the practical application of as early as one hundred years ago and Engels pointed out: mathematics is the study of quantitative relationship between the real world and the spatial form of science. Categories of the study of the modern mathematics has not only confined to the "number" and "shape", mathematical research object can include any form of objective reality and relationships mathematics has deeply penetrated into each discipline [3].

So-called teaching structure which is to point to in certain education ideology, teaching theory and learning theory under the guidance of in the process of teaching activities in a certain environment and the stability of the structure. It will directly reflect the teachers according to what kind of education thought and theory to organize their own teaching activity process which is the education thought. Teaching mode is to carry out teaching activities leopard a plan or model is based on a certain teaching theory and set up a relatively stable teaching activity framework and procedure which is a variety of teaching activities organically connect together to form the dynamic process from the perspective of micro teaching activity. Because mathematics discipline characteristic, mathematics education formed in the course of long-term development is given priority to with the classical theory of education only the cultivation of thinking ability of mathematics training on people which does not pay attention to practical application nor do not speak of mathematics application value and scientific value and eventually lead to the university mathematics teaching effect is not satisfactory.

Therefore, we theoretically research the implementation of teaching assistant system based on web development technique for advanced mathematics in this manuscript. The popularization of computer has increased people's cognition of mathematical knowledge and requirements and with a large 
number of powerful mathematical software changed the way of mathematics teaching the advent of method. In the past many complicated mathematical calculation and therefore become simple and easy which reduces the difficulty of learning to better stimulate students' interest in learning mathematics. Many abstract and complex geometries and reluctant to understand math concepts through computer integrating theoretical teaching and experimental teaching demonstration which realize the combination of teaching and learning and it can greatly improve students' ability of problem analysis and understanding practice ability and practice ability of scientific research. In the following sections we will design the system in practice.

\section{The Design and Implementation of the System}

The General Goal and Guideline. Teaching structure, teaching mode and teaching strategies are in three different levels of concept with teaching structure in a more macro level which reflects a certain education teaching theory in the four core elements in the teaching of the role of stable relationship. It does not depend on the specific teaching content and teaching object. Teaching model is the teaching structure in specific subject areas in the embodiment of the teaching process and the same teaching structure in different teaching content, teaching environment and teaching object which are more than can be derived to guide specific learning process of the teaching mode. Teachers should choose teaching media for students' learning to the present forms of teaching media and render the content design and organization, learning situations of related design [4]. Therefore from the teachers to the teaching media is a strong interaction and teaching media for a variety of teaching forms will provide strong support to provide different types of tools for teachers' teaching from teaching media to the teacher is also a strong interaction. Network auxiliary teaching system covers the information display, answering questions after class, homework management, self-test, practice, and other functions. The use of the system broke the traditional teaching of space and time limit, enhance the communication between teachers and students, teaching cost savings by regulate the teaching process, improve the teaching quality and teaching effect, has been well received by the majority of teachers and students. The learning to teach pay equal attention to the structure of teaching, the student is in the open and interactive learning environment which have a lot of by teachers' selection and design and remove redundant information resources which is conducive to the full play of initiative and enthusiasm. Teachers through the teaching content, teaching media and learning activities designed to make the students in the learning process has a lot of autonomy and to ensure its learning does not happen deviation, can get a teacher or expert at the appropriate time.

The Core Technique of Web based System Development. Web based system is the way of releasing information and it is the use of hypertext, hypermedia information with the method of storage and transfer of technology. It can be a variety of information resources organically which has illustrated information integration ability. Mathematics remote auxiliary teaching system is the product of the combination of web technology and database technology and the system software design of the main task is to solve the problem of dynamic interaction between the web and database [5]. The core techniques are shown below. (1) Common Gateway Interface. This is a kind of programming standards which could call an external application is a web server in the parameters of the standard agreement. (2) Application Program Interface. API provided in the form of dynamic link library and resides on a web server. In order to improve the efficiency of programming, the Microsoft provides a high-level programming interface based on API Internet database connector. (3) Java Language. Java is a simple, object-oriented and easy transmission multithreaded execution control and the three dimensional space design language. It to Internet notes the strong interactivity. Java support database visit to rush which can quickly and effectively connect different correlation of database system. According to the actual situation, we use the prototype method to quickly construct a model with little cost. Users (mainly teachers and students) and developers can quickly determine the needs, and then uses the circulation evolution mode of development, the elaboration of the entire model for continuous and the model required properties increase gradually until all the nature of the content. This module has developed into the final product. Domain knowledge, outside of the 
conceptual syntax, is not a necessary component of an ISS. However, it may be the case that a dialogue strategy with access to a full domain model is more effective than one that relies solely on the learnt model. Of user access control is mainly through the study of the control and management of user rights and permissions is to allow users to an object for a certain operation rights and in this system refers to the user to see the right page, or by modify the data. In the following figure 1, we show the general structure and flowchart for our designed system.

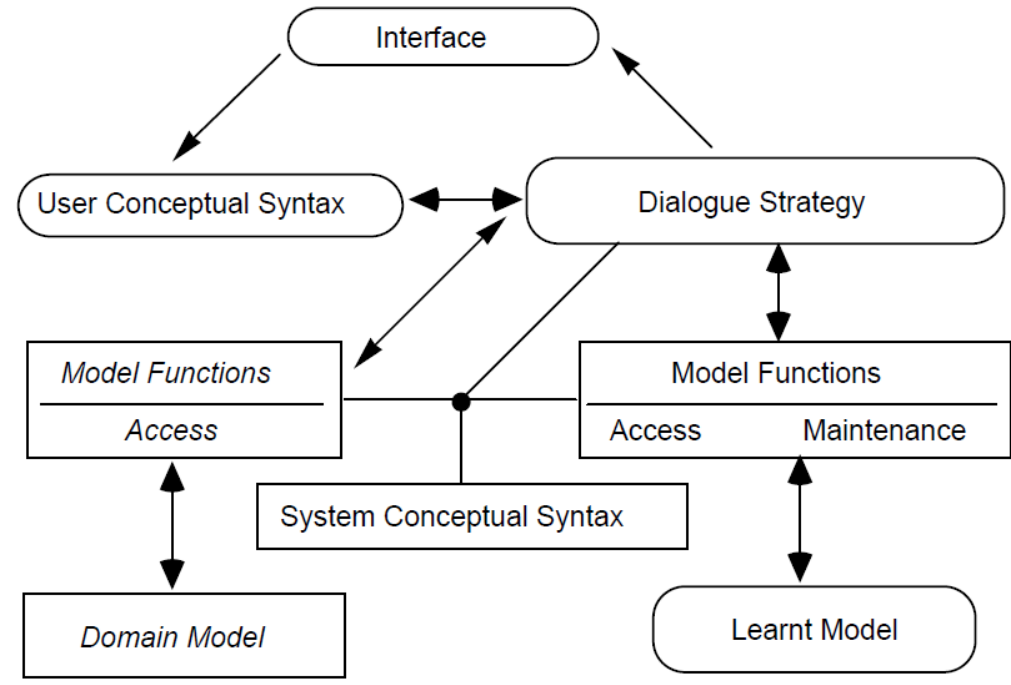

Fig. 1. The General Structure of the Proposed System

Business functionality through Web Service, create the client application invokes the Web Service, make the business functions and the corresponding data into the client, make the customers have a copy directly from the server program and data, can be realized in the network disconnected local data access operations [6].Web services are commonly used protocol through the discrete software components to interact with other software components. Remote clients and applications using the SOAP protocol based on XM L via the HTTP protocol, access to the Web Service. The final Web Service architecture is shown in figure 2.

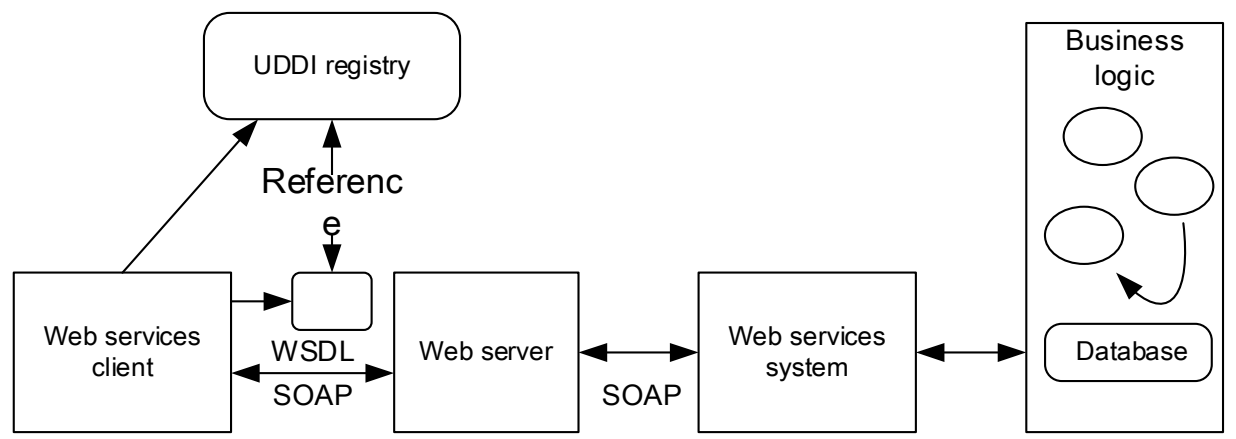

Fig.2. Architecture of Web Service

Due to the use of XML and SOAP, access services, independently of the implementation scheme, therefore, is both Web Service component architecture and the advantages of the Web. Similar to the component development process, Web Service has two characteristics: (1) it has a lookup Service optional registration; (2) it has public interface with clients to invoke the service. In addition, the Web Service also has is better than that of some characteristics of the development of components, such as the use of standard Web protocol to communicate; it can be accessed through the Web; Support is not coupled loose coupling between distributed systems, etc. Web Service has 2 main platforms: the.net and J2EE. The.net platform successfully meet the requirement of Web Service scalable, safe, efficient and portable data and code requirements.

The Code Optimization and General Demonstration. To achieve this kind of access control and allow a user to have multiple roles, a character can be licensed to multiple users a character can 
contain multiple access and a permission can be included more than one role. By the user role has access, it is not directly associated with authority and permissions to access the object operation is achieved by active role. The following XML and mathematical description block describes the relationship of designing the formula of $\int_{a}^{b} \sin (x) e^{x} d x$.

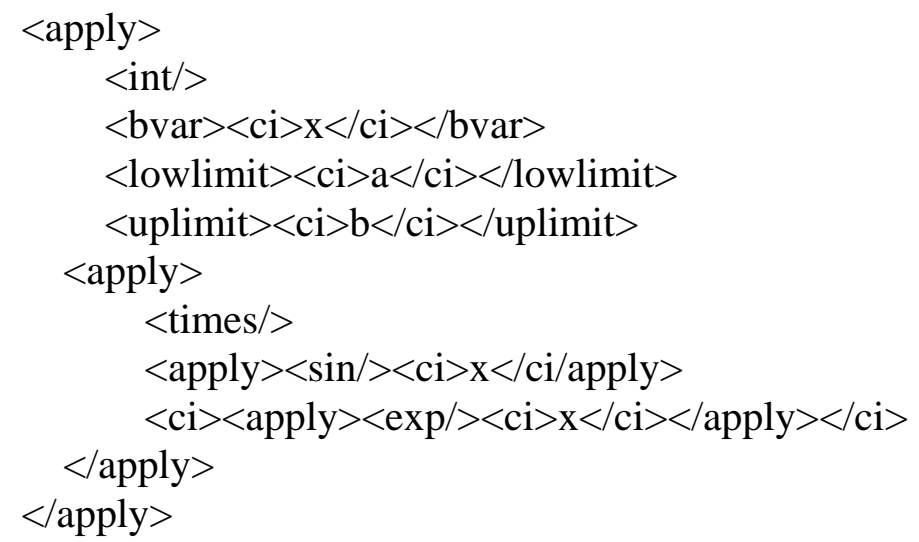

Multilayer distributed application model on J2EE, which can effectively separate the data layer, logic layer and presentation layer. Multilayer structure design is one of the biggest benefits of good ability of extension and load balancing, low coupling between the layers. J2EE architecture allows developers to focus on the business logic of the application. Therefore, J2EE platform can be used to build an extensible system. In the actual application development, especially in the Web application system and if the JSP, Servlet or EJB using JDBC direct access to the data in the database, every data access requests must go through establishing a database connection and open the database to access data and close the database connection and other steps. Therefore, the connection and open the database is a consumer of resources and time-consuming work. If frequent the database operation, the performance of the system will inevitably fell sharply even lead to system crash. Database connection pool technology is the most commonly used method to solve this problem.

In the network auxiliary teaching system has three characters: teacher, student, educational administrators. Teachers have released upload teaching resources, teaching information release homework, review homework, answer questions, question library management, management practice question bank self-test permissions. Students have a browse and download announcement released download information and teaching resources, teachers work, submit the completed homework and check your homework grades and comment on information, browse the library, ask questions, such as the test practice permissions. Educational administrators have released information on teaching, teacher/student information management, set up and modify the system password, and other functions. When students have problems in the learning process and may at any time to access the system of automatic question-answering subsystem. In the teaching design stage, the teacher will be the subject of the most common problems and answers according to certain organization goes into the knowledge base of the course. When students encounter problems, remote submit a description of the problem, through the network system is described according to the problems students submit to search the knowledge base and in accordance with the relevant retrieve content level of high and low which will answer to the problem presented to students. When not in the knowledge base retrieval to answer to the question, the system will notify the student, and will be put to solve the problem of the problem by the teachers responsible for answering questions to answer and add the question and the answer to the knowledge base in the course. The system user interface will be shown in the next section.

The User Interface and Appreciation of the System. To achieve the goal of building the system with better user experience, we modify the user interface through software. In the following figure, we show the UI of our designed system. 


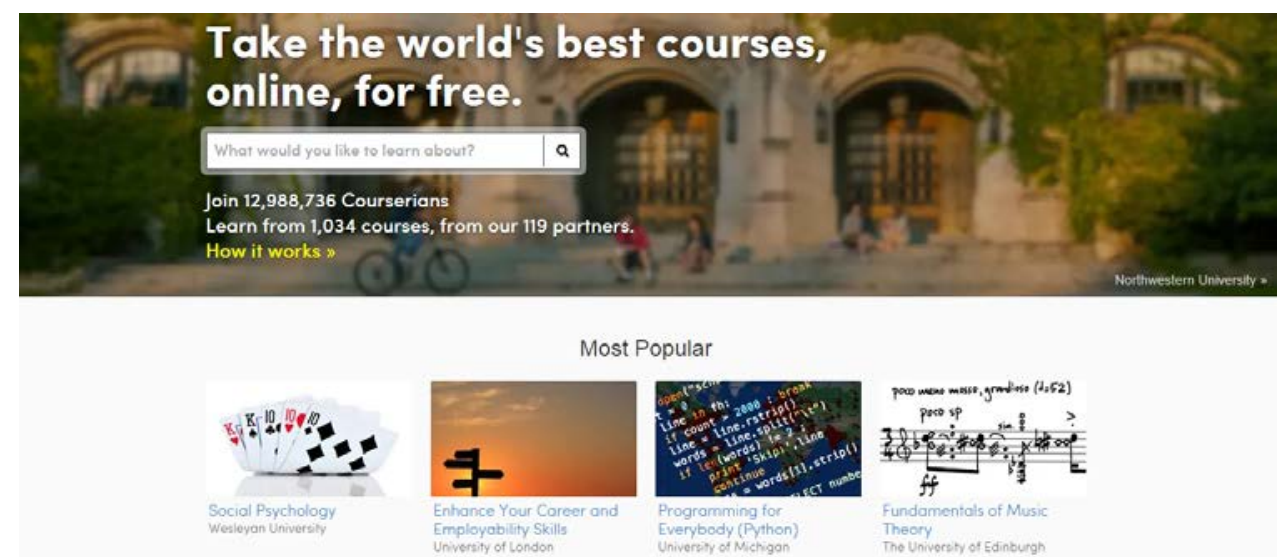

Fig. 3.The User Interface of the Designed System

\section{Conclusion and Summary}

In this paper, we theoretically research the implementation of teaching assistant system based on web development technique for advanced mathematics in this manuscript. It will directly reflect the teachers according to what kind of education thought and theory to organize their own teaching activity process which is the education thought. Teaching mode is to carry out teaching activities leopard a plan or model is based on a certain teaching theory and set up a relatively stable teaching activity framework and procedure. We believe that our designed system will assist the advanced mathematical teachers to perform better when teaching.

\section{References}

[1] El-Dahshan E S A, Hosny T, Salem A B M. Hybrid intelligent techniques for MRI brain images classification[J]. Digital Signal Processing, 2010, 20(2): 433-441.

[2] Lockwood S A, Miller A J, Cromie M M. Preparing Future Biology Faculty: An Advanced Professional Development Program for Graduate Students[J]. American Biology Teacher, 2014.

[3] Gregory J K, Lachman N, Camp C L, et al. Restructuring a basic science course for core competencies: An example from anatomy teaching[J]. Medical teacher, 2009, 31(9): 855-861.

[4] Hwang G, Hung P, Chen N, et al. Mindtool-Assisted In-Field Learning (MAIL): An Advanced Ubiquitous Learning Project in Taiwan[J]. Educational Technology \& Society, 2014, 17(2):4-16.

[5] Yu F Y. Scaffolding student-generated questions: Design and development of a customizable online learning system[J]. Computers in Human Behavior, 2009, 25(5): 1129-1138.

[6] Uemura $\mathrm{K}$, Inagaki $\mathrm{M}$, Zheng $\mathrm{C}$, et al. A novel technique to predict pulmonary capillary wedge pressure utilizing central venous pressure and tissue Doppler tricuspid/mitral annular velocities[J]. Heart \& Vessels, 2014. 